eCommons@AKU

August 2010

\title{
Blastocystis hominis and Dientamoeba fragilis in patients fulfilling irritable bowel syndrome criteria.
}

javed Yakoob

Aga Khan University, javed.yakoob@aku.edu

Wasim Jafri

Aga Khan University, wasim.jafri@aku.edu

Mohammad Asim Beg,

Aga Khan University

Z Abbas

Aga Khan University, zaigham.abbas@aku.edu

Shagufta Naz,

Aga Khan University

See next page for additional authors

Follow this and additional works at: http://ecommons.aku.edu/

pakistan_fhs_mc_med_gastroenterol

Part of the Gastroenterology Commons

\section{Recommended Citation}

Yakoob, j., Jafri, W., Beg, M. A., Abbas, Z., Naz,, S., Islam, M., Khan, R. (2010). Blastocystis hominis and Dientamoeba fragilis in patients fulfilling irritable bowel syndrome criteria.. Parasitology research., 107(3), 679-684.

Available at: http://ecommons.aku.edu/pakistan_fhs_mc_med_gastroenterol/101 


\section{Authors}

javed Yakoob; Wasim Jafri; Mohammad Asim Beg,; Z Abbas; Shagufta Naz,; Muhammad Islam; and Rustam Khan 


\title{
Blastocystis hominis and Dientamoeba fragilis in patients fulfilling irritable bowel syndrome criteria
}

\author{
Javed Yakoob • Wasim Jafri • Mohammad Asim Beg • \\ Zaigham Abbas $\cdot$ Shagufta $\mathrm{Naz} \cdot$ Muhammad Islam • \\ Rustam Khan
}

Received: 17 April 2010 / Accepted: 7 May 2010 / Published online: 8 June 2010

(C) Springer-Verlag 2010

\begin{abstract}
Studies have suggested a possible role for Blastocystis hominis and Dientamoeba fragilis in the etiology of irritable bowel syndrome (IBS). We studied the prevalence of $B$. hominis and $D$. fragilis in patients with IBS-diarrhea (IBS-D). Three hundred and thirty patients were enrolled, 171 (52\%) with IBS-D and 159 (48\%) were controls, respectively. Stool microscopy, culture, and polymerase chain reaction (PCR) for B. hominis and $D$. fragilis were done. B. hominis was positive by stool microscopy in $49 \%(83 / 171)$ of IBS compared to $24 \%$ $(27 / 159)$ in control $(p<0.001)$. B. hominis culture was positive in $53 \%(90 / 171)$ in IBS compared to $16 \%(25 / 159)$ in control $(p<0.001)$. B. hominis PCR was positive in $44 \%$ (75/171) in IBS compared to $21 \%(33 / 159)$ in control $(p<$ $0.001)$. D. fragilis microscopy was positive in $3.5 \%(6 / 171)$ in IBS-D compared to $0.6 \%(1 / 159)$ in control $(p=0.123)$. D. fragilis culture was positive in $4 \%(7 / 171)$ in IBS compared to $1.3 \%(2 / 159)$ in control $(p=0.176)$. D. fragilis PCR was positive in $4 \%(6 / 171)$ in IBS-D compared to $0 \%$ $(0 / 159)$ in control $(p=0.030)$. B. hominis is common, while $D$. fragilis was less prevalent in our patients with IBS-D. $B$. hominis and $D$. fragilis culture had a better yield compared to stool microscopy and PCR.
\end{abstract}

M. A. Beg

Department of Microbiology and Pathology,

The Aga Khan University,

Karachi, Pakistan

J. Yakoob $(\bowtie) \cdot$ W. Jafri $\cdot$ Z. Abbas $\cdot$ S. Naz $\cdot$ M. Islam $\cdot$ R. Khan Department of Medicine, Aga Khan University Hospital,

Stadium Road,

Karachi 74800, Pakistan

e-mail: yakoobjaved@hotmail.com

\section{Introduction}

Parasitic infections afflict a wide range of populations in both urban and rural areas throughout the world. The direct impact of protozoan parasites on human is considerable in the third world countries with poor sanitation and quality of drinking water (Zonta et al. 2010). Parasitic infections such as Blastocystis hominis and Dientamoeba fragilis are associated with abdominal pain, bloating, and alteration of bowel habits resembling irritable bowel syndrome (IBS; Yakoob et al. 2004; Johnson et al. 2004). However, a diagnosis of IBS is based on ROME III criteria independently of specific stool collection and testing methods recommended for detection of parasites (Drossman 2006). Also, the methods commonly used to diagnose parasitic infections have a poor yield, and hence, these infections are never diagnosed. The prevalence of these parasites is highest in areas of poor sanitation and drinking water treatment as they are transmitted by fecal-oral route.

A significantly increased level of IgG2 levels against $B$. hominis was found in IBS patients compared with asymptomatic controls, indicating that the predominant response in these patients may be directed to carbohydrate antigens (Hussain et al. 1997). A number of studies have incriminated D. fragilis as a cause of IBS, allergic colitis, and diarrhea in human immunodeficiency virus patients (Johnson et al. 2004). In a recent study, a high prevalence of $D$. fragilis was reported in fecal samples collected from patients attending complementary medicine practitioners in the British Isles (Windsor et al. 2002). Approximately 25\% of these stated intestinal symptoms, ranging from acute gastroenteritis to chronic intestinal symptoms, while similar in proportion did not report having intestinal symptoms. It is also noteworthy that over half of the $D$. fragilis positive samples were found in combination with $B$. hominis as 
previously observed (Stensvold et al. 2007; Schuster and Jackson 2009; Stark et al. 2005; Norberg et al. 2003). B. hominis is a common human intestinal parasite that is commonly found in stool on examination. It can be easily demonstrated by light microscopy, culture, and polymerase chain reaction (PCR) examination of stool samples (Norberg et al. 2003; Zaman and Khan 1994). The pathogenic role of $B$. hominis is still controversial as it is frequently found not only in individuals with enteric symptoms but also in apparently healthy and asymptomatic subjects (Silberman et al. 1996). It is thought that genotypic difference exists between the asymptomatic and symptomatic B. hominis isolates (Stensvold et al. 2006; Yoshikawa et al. 2000). The aim of this study was to determine the prevalence of $B$. hominis and D. fragilis in patients with symptoms of IBS-D and compared them with healthy immunocompetent controls.

\section{Materials and methods}

\section{Patient}

A total of 330 stool samples were examined that were obtained from patients fulfilling Rome III criteria of IBS-D in $171(52 \%)$ and $159(48 \%)$ controls who attended the gastroenterology outpatient clinic at the Aga Khan University, Karachi between January 2008 and December 2009, respectively (Drossman 2006). The mean age of patients with IBS-D was $40 \pm 15$, age range $16-83$ years, and male: female ratio of 117:54. In control group, 159 (48\%) were healthy volunteers with mean age $42 \pm 14$. These patients underwent thorough history, physical examination, complete blood count, serum creatinine, electrolytes, and stool microscopy, culture, and PCR for B. hominis and $D$. fragilis. These patients had intact immunity. The study was approved by institutional ethics review committee.

All the stool specimens were processed by stool microscopy for $B$. hominis and $D$. fragilis. Stool culture was done for $B$. hominis and $D$. fragilis. DNA was extracted from the unfixed stool specimen, and it was used for PCR for B. hominis and D. fragilis. A note was made of the presence of other parasites such as Giardia lamblia, Entamoeba species, and Cryptosporidium, and these patients, ten in number, were excluded while no patient demonstrated Enterobius vermicularis. In previous study, $D$. fragilis mono-nucleated or bi-nucleated forms have been documented in the lumen of E. vermicularis (Johnson et al. 2004). A microbiological investigation was also performed to detect Salmonella spp., Campylobacter jejuni, Clostridium difficile, and Vibrio cholerae. However, a viral screen was not carried out on stool specimens in view of restriction of cost.

\section{Microscopy of fecal smear}

Fecal sample microscopy for demonstrating B. hominis was done as described before (Zaman and Khan 1994); approximately $2 \mathrm{mg}$ of feces was emulsified on a glass slide in one drop of physiologic saline and covered with a cover slip. A similar preparation was made on another slide using Lugol's iodine. These preparations were examined under both the low power $(\times 10)$ and high dry $(\times 40)$ objectives. Specimen smear was fixed in sodium acetate/acetic acid/formalin (SAF) and then stained with modified trichrome stain to look for D. fragilis (Johnson et al. 2004).

\section{Culture}

Jones medium without starch was used for culturing $B$. hominis as described before (Zaman and Khan 1994). The cultures were incubated at $37^{\circ} \mathrm{C}$ and examined after $48 \mathrm{~h}$. If no $B$. hominis were seen up to further 2 days, they were regarded as negative. The sediment was examined under both the low power $(\times 10)$ and high dry $(\times 40)$ objectives. D. fragilis culture was done in Robinson's medium as previously described (Windsor et al. 2003).

\section{Extraction of genomic DNA}

Stool DNA was extracted by using Stool DNA Extraction kit (Qiagen) according to the manufacturer's protocol. Extracted DNA was stored at $-20^{\circ} \mathrm{C}$ until PCR was carried out for B. hominis and D. fragilis

\section{PCR}

The primers used were previously described (Table 1). The primer pair SR1F and SR1R was used to amplify a conserved region of the $B$. hominis small subunit (SSU) rRNA, while primer TRD3 and TRD5 design was based on the sequence of the of SSU rRNA gene of D. fragilis (Silberman et al. 1996; Yoshikawa et al. 2000; Table 1). The PCR reaction volume was $25 \mu$ l that comprised of $2.5 \mu \mathrm{l}$ of $10 \times$ PCR buffer (Promega), $2.0 \mu \mathrm{l}$ of $25 \mathrm{mM}$ $\mathrm{MgCl}_{2}$ (Promega), $0.4 \mu \mathrm{l}$ of deoxyribo-nucleotide triphosphate mix (10 mM each dNTP, Promega), $0.5 \mu \mathrm{l}(5 \mathrm{IU} / \mu \mathrm{l})$ of Taq polymerase (Promega), $1 \mu \mathrm{l}(0.25 \mu \mathrm{M})$ primers (IDT), and $2.0 \mu \mathrm{l}$ of template DNA. The PCR products and molecular markers were electrophoresed in $2 \%$ agarose gel with Tris-acetate-EDTA electrophoresis buffer. The size markers were $100 \mathrm{bp}$ ladder (Promega, USA). The PCR amplification for each primer pair was repeated at least thrice. Bands were visualized by the imaging system (Gel Doc 2000, Gel Documentation System, Bio Rad, UK) after being stained with ethidium bromide. 
Table 1 Primer sequences used for Blastocystis hominis and Dientamoeba fragilis

\begin{tabular}{|c|c|c|c|c|}
\hline & & Primer sequences $5^{\prime}-3^{\prime}$ & Amplified product size, bp & PCR cycles \\
\hline Blastocystis hominis & & & \multirow{3}{*}{1,800} & \multirow{3}{*}{$\begin{array}{l}94^{\circ} \mathrm{C} \text { for } 3 \mathrm{~min} ; 94^{\circ} \mathrm{C} \text { for } 30 \mathrm{~min}, 57^{\circ} \mathrm{C} \\
\text { for } 1 \min 30 \mathrm{~s}, 72^{\circ} \mathrm{C} \text { for } 2 \min (35 \text { cycles }) \\
72^{\circ} \mathrm{C} 7 \mathrm{~min}\end{array}$} \\
\hline $\mathrm{SR} 1^{\mathrm{a}}$ & $\mathrm{F}$ & $\begin{array}{l}\text { GCT TAT CTG GTT GATCCT } \\
\text { GCC AGT AGT }\end{array}$ & & \\
\hline SR2 & $\mathrm{R}$ & $\begin{array}{l}\text { TGA TCC TTC CGC AGG } \\
\text { TTC ACC TA }\end{array}$ & & \\
\hline Dientamoeba fragilis & & & \multirow{3}{*}{1,700} & \multirow{3}{*}{$\begin{array}{l}94^{\circ} \mathrm{C} \text { for } 5 \mathrm{~min} ; 94^{\circ} \mathrm{C} \text { for } 1 \mathrm{~min}, 56^{\circ} \mathrm{C} \\
\text { for } 1 \min 30 \mathrm{~s}, 72^{\circ} \mathrm{C} \text { for } 2 \min (35 \text { cycles }) \text {; } \\
72^{\circ} \mathrm{C} 5 \mathrm{~min}\end{array}$} \\
\hline $\mathrm{TRD}^{\mathrm{b}}$ & $\mathrm{F}$ & GATCCAACGGCAGGTTCACCTACC & & \\
\hline TRD5 & $\mathrm{R}$ & GATACTTGGTTGATCCTGCCAAGG & & \\
\hline
\end{tabular}

${ }^{\text {a }}$ Yoshikawa et al. (2000)

${ }^{\mathrm{b}}$ Silberman et al. (1996)

Statistical method

Results are expressed as mean \pm standard deviation for continuous variables (e.g., age) and number (percentage) for categorical data (e.g., gender, stool culture, and diarrhea). Univariate analysis was performed by using the independent sample $t$ test, Pearson Chi-square test, and Fisher exact test where appropriate. A $p$ value of $<0.05$ was considered as statistically significant. All $p$ values were two sided. Statistical interpretation of data was performed by using the computerized software program SPSS version 16.0.

\section{Results}

The age and sex of the patients were not related to the positivity of $D$. fragilis and $B$. hominis when culture was used as the diagnostic test for $D$. fragilis and $B$. hominis. One hundred ninety-three (59\%) had diarrhea, and 71 (52\%) had abdominal pain.

Distribution and diagnostic yield of various tests for B. hominis

B. hominis was positive by microscopy in 33\% (110/330), culture in 35\% (115/330), and PCR in 33\% (108/330; Table 2). Microscopy for B. hominis was positive in $76 \%$ $(83 / 110)$ in patients fulfilling the criteria of IBS compared to $24 \%(27 / 110)$ in control $(p<0.001$; Table 3$)$. Culture for B. hominis was positive in $78 \%(90 / 115)$ in IBS compared to $22 \%(25 / 115)$ in control $(p<0.001)$. PCR for B. hominis was positive in $69 \%(75 / 108)$ in IBS compared to $31 \%(33 /$ $108)$ in control $(p<0.001$; Table 3$)$.

Distribution and diagnostic yield of various tests for $D$. fragilis

D. fragilis was positive by microscopy in $2 \%(7 / 330)$, culture in $3 \%(11 / 330)$, and PCR in $2 \%(6 / 330)$, respectively (Fig 1; Table 2). Microscopy for $D$. fragilis was positive in $86 \%(6 / 7)$ in IBS compared to $14 \%(1 / 7)$ in control ( $p=0.123$; Table 3). Culture for $D$. fragilis was positive in $64 \%(7 / 11)$ in IBS compared to $36 \%(4 / 11)$ in control $(p=0.425$; Table 3$)$. PCR for $D$. fragilis was positive in $86 \%(6 / 6)$ in IBS compared to none in control $(p=0.030$; Table 3).

Coinfection with $B$. hominis and D. fragilis in different groups

Microscopy was positive for both $B$. hominis and $D$. fragilis in $3 \%(5 / 171)$ with IBS compared to zero $(0 / 159)$ in control $(p=0.061$; Table 2). Culture for both $B$. hominis and $D$. fragilis was positive in $2.3 \%(4 / 171)$ with IBS compared to zero $(0 / 159)$ in control $(p=0.123$; Table 3$)$. PCR was positive for both $B$. hominis and $D$. fragilis in $3 \%(5 / 171)$ with IBS compared to zero $(0 / 159)$ in control $(p=0.061$; Table 3$)$.

Comparison of culture for $B$. hominis and D. fragilis with other test

In patients with IBS, $B$. hominis culture was positive in 90 , while microscopy for $B$. hominis was positive in $82 \%$ (74/ $90 ; p<0.001)$ and PCR in $76 \%(68 / 90 ; p<0.001)$. In IBS patients, $D$. fragilis was positive in seven (4\%) with culture,

Table 2 Diagnostic yield of various tests for Blastocystis hominis and Dientamoeba fragilis

\begin{tabular}{lccr}
\hline & Microscopy & Culture & PCR \\
\hline $\begin{array}{l}\text { Blastocystis hominis } \\
\text { Positive }\end{array}$ & $110(33)$ & $115(35)$ & $108(33)$ \\
Negative & $220(67)$ & $215(65)$ & $222(67)$ \\
$\begin{array}{l}\text { Dientamoeba fragilis } \\
\text { Positive }\end{array}$ & $7(2)$ & $11(3)$ & $6(2)$ \\
Negative & $323(98)$ & $319(97)$ & $324(98)$ \\
Blastocystis hominis & and Dientamoeba fragilis & \\
Positive & $5(2)$ & $4(1)$ & $5(2)$ \\
Negative & $325(98)$ & $326(99)$ & $325(98)$ \\
\hline
\end{tabular}


Table 3 Comparison of Blastocystis hominis and Dientamoeba fragilis in different groups

\begin{tabular}{|c|c|c|c|}
\hline & IBS, $n=171$ & Control, $n=159$ & $P$ value \\
\hline \multicolumn{4}{|c|}{ Blastocystis hominis } \\
\hline \multicolumn{4}{|l|}{ Microscopy } \\
\hline Positive & $83(48)$ & $27(17)$ & \multirow[t]{2}{*}{$<0.001$} \\
\hline Negative & $88(52)$ & $132(83)$ & \\
\hline \multicolumn{4}{|l|}{ Culture } \\
\hline Positive & $90(53)$ & $25(16)$ & \multirow[t]{2}{*}{$<0.001$} \\
\hline Negative & $81(47)$ & $134(84)$ & \\
\hline \multicolumn{4}{|l|}{ PCR } \\
\hline Positive & $75(44)$ & $33(21)$ & \multirow[t]{2}{*}{$<0.001$} \\
\hline Negative & $96(56)$ & $126(79)$ & \\
\hline \multicolumn{4}{|c|}{ Dientamoeba fragilis } \\
\hline \multicolumn{4}{|l|}{ Microscopy } \\
\hline Positive & $6(4)$ & $27(17)$ & \multirow[t]{2}{*}{0.123} \\
\hline Negative & $165(96)$ & $132(83)$ & \\
\hline \multicolumn{4}{|l|}{ Culture } \\
\hline Positive & $7(4)$ & $4(2)$ & \multirow[t]{2}{*}{0.545} \\
\hline Negative & $164(96)$ & $155(98)$ & \\
\hline \multicolumn{4}{|l|}{ PCR } \\
\hline Positive & $6(4)$ & $0(0)$ & \multirow[t]{2}{*}{0.030} \\
\hline Negative & $165(96)$ & $159(100)$ & \\
\hline \multicolumn{4}{|c|}{$\begin{array}{l}\text { Coinfection with } \\
\text { B. hominis and } \\
\text { Dientamoeba fragilis }\end{array}$} \\
\hline \multicolumn{4}{|l|}{ Microscopy } \\
\hline Positive & $5(3)$ & $0(0)$ & \multirow[t]{2}{*}{0.061} \\
\hline Negative & $166(97)$ & $159(100)$ & \\
\hline \multicolumn{4}{|l|}{ Culture } \\
\hline Positive & $4(2)$ & $0(0)$ & \multirow[t]{2}{*}{0.124} \\
\hline Negative & $167(98)$ & $159(100)$ & \\
\hline \multicolumn{4}{|l|}{ PCR } \\
\hline Positive & $5(3)$ & $0(0)$ & \multirow[t]{2}{*}{0.061} \\
\hline Negative & $166(97)$ & $159(100)$ & \\
\hline
\end{tabular}

while microscopy for $D$. fragilis was positive in 57\% (4/7; $p<0.001)$, and PCR for $D$. fragilis was not positive in any patients $0 / 7(p=1)$.

\section{Discussion}

This study showed $B$. hominis was significantly positive in IBS while $D$. fragilis was positive in only $4 \%$. In patients with IBS, B. hominis culture was significantly positive compared to microscopy and PCR for B. hominis. Similarly, culture of $D$. fragilis had a better yield compared to microscopy with modified trichrome staining and PCR. Coinfection with both $B$. hominis and $D$. fragilis in IBS patients was documented in $3 \%$ with culture and $2.3 \%$ by microscopy. B. hominis and $D$. fragilis infections did not

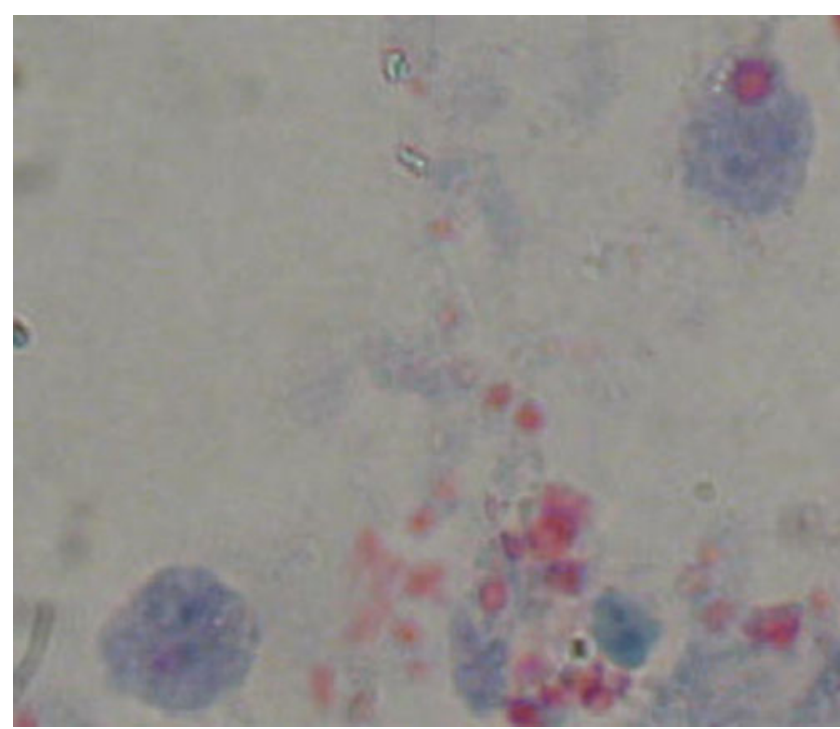

Fig. 1 Dientamoeba fragilis in stool specimen stained with modified trichrome stain under $\times 100$ magnification

show any age and gender distribution. However, one third of the $D$. fragilis infections were in patients who were in their twenties. None of the patients with $D$. fragilis demonstrated concomitant infection with $E$. vermicularis.

The implications of this study are that $B$. hominis are found with a higher frequency in our IBS patients compared to $D$. fragilis. The coinfection of $B$. hominis and $D$. fragilis was seen in only IBS group and none in the fecal samples from control group comprising of healthy individuals without gastrointestinal symptoms. This study has shown that $D$. fragilis was associated with IBS-like symptoms in our patients, though it has a low prevalence. It is still possible that we were not able to document all the $D$. fragilis infections in spite of using three different modalities, i.e., microscopy with modified trichrome staining of fixed stool specimen, culture of stool specimens, and PCR examination of unfixed stool specimens. It is possible that if we examined three fixed and three unfixed stool specimens from each of these patients, the yield might have increased. Also, increasing number of symptomatic patients would have helped. Windsor et al. found D. fragilis culture has the best yield compared to microscopy with modified iron-hematoxylin staining of stool specimen (Windsor et al. 2003). An Australian study previously described an association between $D$. fragilis and IBS with 21 IBS patients diagnosed with concurrent $D$. fragilis infection (Brody et al. 2002). In another prospective study, 6,750 fecal samples were examined for $D$. fragilis. Trophozoites of $D$. fragilis were detected in $60(0.9 \%)$ patients by permanent staining, using a modified iron-hematoxylin stain that was confirmed by PCR (Stark et al. 2005).

Chronic symptoms were present in $32 \%$ with diarrhea and abdominal pain present in all of these patients (Stark et 
al. 2005). In the control group comprising of 900 stool samples from patients without gastrointestinal symptoms, no $D$. fragilis was detected by permanent staining (Stark et al. 2005). Our results concur with that of Stark et al. (2005). This is the first report of documentation of $D$. fragilis in our population. It was found infrequently in our patients with IBS. D. fragilis is a pathogenic organism rather than a commensal, as diarrhea and abdominal pain were the most common symptoms in both acute and chronic infections (Johnson et al. 2004; Stark et al. 2005; Dobell 1940; Windsor and Johnson 1999; Yang and Scholten 1977). We also did not find any correlation between $D$. fragilis and $E$. vermicularis, a proposed vector of transmission for $D$. fragilis in keeping with previous study (Stark et al. 2005).

The results of this study concur with a previous study that culture of $B$. hominis has a better yield than that of microscopy (Zaman and Khan 1994). However, B. hominis culture yield was not significantly different from $B$. hominis PCR. In recent years, studies have suggested that there are genotypes of $B$. hominis associated with symptomatic and asymptomatic states (Hussein et al. 2008; Tan 2008; Yan et al. 2006). Different degrees of pathological changes were present among infected rats by symptomatic subtypes 1, 3, and 4 compared with asymptomatic subtypes, e.g., 2, 3, and 4 (Hussein et al. 2008). The moderate and severe degree of pathological changes was found only in symptomatic subtypes infected rats, while mild degree was found only in asymptomatic subtypes infected rats (Hussein et al. 2008). The intestinal cell permeability was increased in symptomatic subtype 1 compared to symptomatic subtypes, e.g., 3 and 4, infected rats (Hussein et al. 2008). Minimal effects were found in the asymptomatic and control groups. These results proved that subtype 1 was clinically and statistically highly relevant to the pathogenicity of $B$. hominis, while subtype 2 was irrelevant (Hussein et al. 2008). Also, the results suggested the presence of pathogenic and nonpathogenic strains among subtypes 3 and 4 (Hussein et al. 2008). A study from China also described a possible relationship between subtype 1 and a pathogenic potential of $B$. hominis (Yan et al. 2006). Similarly, Blastocystis subtype 3 was described as the most dominant genotype in asymptomatic individual, and subtype 1 determined all of symptomatic patients in studies from Turkey and Pakistan (Eroglu et al. 2009; Yakoob et al. 2010). D. fragilis prevalence appears to be low in our IBS population.

In conclusion, we demonstrated presentation of $B$. hominis and $D$. fragilis infections with symptoms of IBS; however, only a prospective study with a larger sample size is required to confirm this association of $D$. fragilis with IBS-like symptoms in our population.

Acknowledgements This study was supported by the Higher Educational Commission Grant Ref: $20-774 / R \& D / 06 / 267$ to JY. We are thankful to Dr. C. Graham Clark (Department of Infectious and Tropical Diseases, London School of Hygiene and Tropical Medicine, London, England) for providing us the controlled DNA of D. fragilis cultures and to Dr. JJ Windsor (NPHS Microbiology Aberystwyth, Bronglais Hospital, Ceredigion, UK) for his valuable comments.

Conflict of interest The authors declare they have no conflict of interests.

\section{References}

Brody TJ, Warren E, Wettstein A, Robertson P, Recabarren A (2002) Eradication of Dientamoeba fragilis can resolve IBS-like symptoms. J Gastroenterol Hepatol 17:A103

Dobell C (1940) Researches on the intestinal protozoa of monkeys and man. The life history of Dientamoeba fragilis: observations, experiments and speculations. Parasitology 32:417-461

Drossman DA (2006) Rome III: the new criteria. Chin J Dig Dis 7:181-185

Eroglu F, Genc A, Elgun G, Koltas IS (2009) Identification of Blastocystis hominis isolates from asymptomatic and symptomatic patients by PCR. Parasitol Res 105:1589-1592

Hussein EM, Hussein AM, Eida MM, Atwa MM (2008) Pathophysiological variability of different genotypes of human Blastocystis hominis Egyptian isolates in experimentally infected rats. Parasitol Res 102:853-860

Hussain R, Jafri W, Zuberi S, Baqai R, Abrar N, Ahmed A, Zaman V (1997) Significantly increased IgG2 subclass antibody levels to Blastocystis hominis in patients with irritable bowel syndrome. Am J Trop Med Hyg 56:301-306

Johnson EH, Windsor JJ, Clark CG (2004) Emerging from obscurity: biological, clinical, and diagnostic aspects of Dientamoeba fragilis. Clin Microbiol Rev 17:553-570

Norberg A, Nord CE, Evengard B (2003) Dientamoeba fragilis-a protozoal infection which may cause severe bowel distress. Clin Microbiol Infect 9:65-68

Schuster H, Jackson RS (2009) Prevalence of Dientamoeba fragilis among patients consulting complementary medicine practitioners in the British Isles. J Clin Pathol 62:182-184

Silberman JD, Clark CG, Sogin ML (1996) Dientamoeba fragilis shares a recent common evolutionary history with the trichomonads. Mol Biochem Parasitol 76:311-314

Stark D, Beebe N, Marriott D, Ellis J, Harkness J (2005) Prospective study of the prevalence, genotyping and clinical relevance of Dientamoeba fragilis infections in an Australian population. $\mathrm{J}$ Clin Microbiol 43:2718-2723

Stensvold CR, Brillowska-Dabrowska A, Nielsen HV, Arendrup MC (2006) Detection of Blastocystis hominis in unpreserved stool specimens by using polymerase chain reaction. J Parasitol 92:1081-1087

Stensvold CR, Arendrup MC, Mølbak K, Nielsen HV (2007) The prevalence of Dientamoeba fragilis in patients with suspected enteroparasitic disease in a metropolitan area in Denmark. Clin Microbiol Infect 13:839-842

Tan KSW (2008) New insights on classification, identification and clinical relevance of Blastocystis spp. Clin Microbiol Rev 21:639665

Windsor JJ, Johnson EH (1999) Dientamoeba fragilis: the unflagellated human flagellate. Br J Biomed Sci 56:293-306

Windsor JJ, Macfarlane L, Hughes-Thapa G, Jones SK, Whiteside TM (2002) Incidence of Blastocystis hominis in faecal samples submitted for routine microbiological analysis. $\mathrm{Br} \mathrm{J}$ of Biomed Sc 59:154-157 
Windsor JJ, Macfarlane L, Hughes-Thapa G, Jones SK, Whiteside TM (2003) Detection of Dientamoeba fragilis by culture. $\mathrm{Br} \mathrm{J}$ Biomed Sci 60:79-83

Yakoob J, Jafri W, Jafri N, Khan R, Islam M, Beg MA, Zaman V (2004) Irritable bowel syndrome: in search of an etiology: role of Blastocystis hominis. Am J Trop Med Hyg 70:383-385

Yakoob J, Jafri W, Beg MA, Abbas Z, Naz S, Islam M, Khan R (2010) Irritable bowel syndrome: is it associated with genotypes of Blastocystis hominis. Parasitol Res 106:10331038

Yan Y, Su S, Ye J, Lai R, Liao H, Ye J, Li X, Luo X, Chen G (2006) Genetic variability of Blastocystis hominis isolates in China. Parasitol Res 99:597-601
Yang J, Scholten TH (1977) Dientamoeba fragilis: a review with notes on its epidemiology, pathogenicity, mode of transmission and diagnosis. Am J Trop Med Hyg 26:16-22

Yoshikawa H, Abe N, Iwasawa M, Kitano S, Nagano I, Wu Z, Takahashi (2000) Genomic analysis of Blastocystis hominis strains isolated from two long-term Health care facilities. J Clin Microbiol 38:1324-1330

Zaman V, Khan K (1994) A comparison of direct microscopy with culture for the diagnosis of Blastocystis hominis. Southeast Asian J Trop Med Hyg Public Health 25:792-793

Zonta ML, Oyhenart EE, Navone GT (2010) Nutritional status, body composition, and intestinal parasitism among the Mbyá-Guaraní communities of Misiones, Argentina. Am J Hum Biol 22:193-200 